\title{
High Average-Efficiency Multimode RF Transmitter Using a Hybrid Quadrature Polar Modulator
}

\author{
Chien-Jung Li, Student Member, IEEE, Chi-Tsan Chen, Tzyy-Sheng Horng, Senior Member, IEEE, \\ Je-Kuan Jau, Member, IEEE, and Jian-Yu Li
}

\begin{abstract}
This paper presents a hybrid quadrature polar modulator (HQPM) to drive the power amplifier (PA) highly efficiently in a wireless RF transmitter required for multimode operation. For enhancing the transmit efficiency, a switching-mode $P A$ realized as Class-E design is used in the transmitter. The HQPM consists of a quadrature modulator for processing the RF modulated carrier and a Class-S modulator for processing the supply-voltage signal. The quadrature modulator and the Class-S modulator deliver the output signals with proportional envelope variation before being inserted into the $R F$-input terminal and the supply-voltage terminal of a Class-E PA, respectively, causing the double envelope modulation to distort the modulated RF signal at the PA output. Therefore, a digital predistorter is embedded in the HQPM for compensation. The proposed HQPM-based transmitter can help reducing the average dc and input $R F$ powers and the output feedthrough levels so as to enhance power added efficiency and adjacent channel power rejection remarkably.
\end{abstract}

Index Terms-Class-E power amplifier (PA), Class-S modulator, digital predistorter, wireless RF transmitter.

\section{INTRODUCTION}

$\mathbf{T}$ HE conventional quadrature modulator-based transmitter, as illustrated in Fig. 1(a), relies on a linear power amplifier $(\mathrm{PA})$ that is most often realized as Class A or $\mathrm{AB}$ design with a constant supply voltage $\left(V_{s}=\right.$ constant). In such types of architecture, power back-off is commonly used to prevent the PA nonlinear effects at the cost of sacrifice in PA efficiency. Various techniques such as dynamic bias [1], [2] and envelope tracking [3], [4] have been developed to improve efficiency in a quadrature modulator-based transmitter. These techniques adjust the bias voltage in relation to output power level of the PA. However, the efficiency performance of these techniques is still limited by the linear PAs.

A different approach is to implement a linear transmitter with a highly efficient, but nonlinear, switching-mode PA. Recently, polar modulator [5], [6], as illustrated in Fig. 1(b), has been adopted for the enhanced data rates for global evolution (EDGE) system. It applies the concept of the envelope elimination and restoration (EER) transmitter [7], except when

Manuscript received August 14, 2007; revised October 30, 2007. This work was supported in part by the Ministry of Education under Program of Aim for the Top University Plan, Taiwan, R.O.C., and by the National Science Council, Taiwan, R.O.C., under Grant 95-2221-E-110-027. This paper was recommended by Guest Editor L. Larson.

C.-J. Li, C.-T. Chen, and T.-S. Horng are with the Department of Electrical Engineering, National Sun Yat-Sen University, Kaohsiung 804, Taiwan, R.O.C. (e-mail: jason@ee.nsysu.edu.tw).

J.-K. Jau and J.-Y. Li are with the Information and Communications Laboratories, Industrial Technology and Research Institute, Hsinchu, 310 Taiwan, R.O.C. (e-mail: jekuan@itri.org.tw).

Digital Object Identifier 10.1109/TCSII.2008.918969

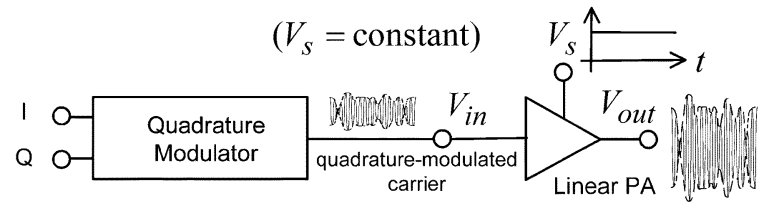

(a)

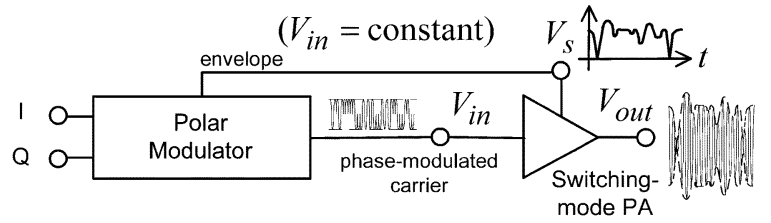

(b)

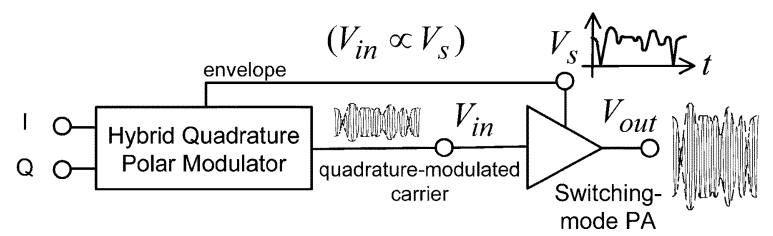

(c)

Fig. 1. Comparison of linear RF transmitter architectures based on different modulator schemes. (a) Quadrature modulator. (b) Polar modulator. (c) Hybrid quadrature polar modulator.

directly generating the envelope and phase signals from a digital baseband processor to improve modulation accuracy after compensating for the delay difference. When a high-efficiency switching-mode PA is used in this architecture, the envelope signal amplitude-modulates the supply voltage of the PA, which reduces the average dc power consumption. The input RF signal contains phase modulation, and maintains a constant amplitude ( $V_{\text {in }}=$ constant $)$ to assure the PA of operating in switching mode. However, maintaining the same high constant amplitude for the input RF signal is disadvantageous to power-added efficiency (PAE) at low output power levels. Besides, the feedthrough from the input RF signal with constant amplitude often contaminates the output modulated waveform near the zero crossing.

To address the drawbacks of the polar modulator-based transmitter architecture, the authors have proposed a hybrid quadrature polar modulator (HQPM) [8], [9]. As illustrated in Fig. 1(c), the modulator can enforce a linear relation between the input RF amplitude and the supply voltage $\left(V_{\text {in }} \propto V_{s}\right)$ in a switchingmode PA. This provides the input RF signal and supply-voltage signal with the same time-varying envelope. This way, the PAE is optimized, because at any transient, the supply voltage follows the same variation of input RF amplitude. In addition, at the transient of the zero-crossing, the input RF signal has zero 


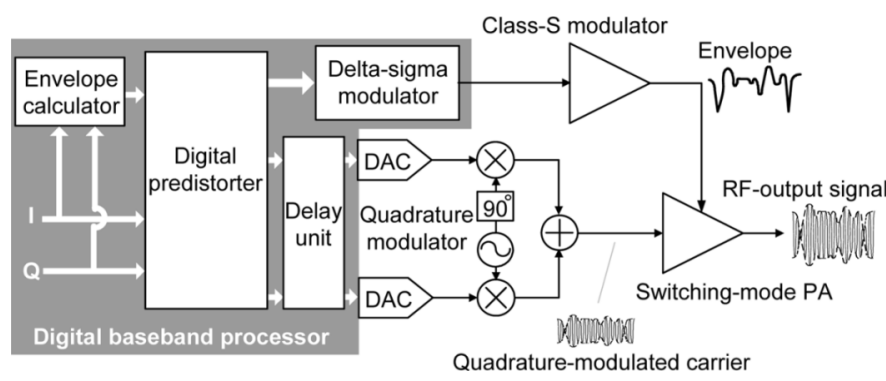

Fig. 2. Block diagram of the HQPM-based transmitter.

amplitude. Although the envelope-varying input RF signal cures the signal feedthrough problem in polar modulation architecture, the signal quality can still be affected by double envelope modulation, since the input RF signal and supply-voltage signal contain the envelope information simultaneously. A digital predistortion technique is applied to overcome this problem. The authors have covered the linearity and efficiency aspects of the proposed transmitter system in [8] and [9], but those treatments were not complete because of lack of mutual dependence between gain and efficiency for the switching-mode PA excited by the HQPM. In this paper, we complete the coverage of this transmitter system by expanding the treatment to characterize the HQPM-excited switching-mode PA with respect to the linearized gain and PAE.

An advantage of the HQPM-based transmitter is that it can apply large amounts of digital techniques, which allows the transmitter to have the potential to attain different modulation requirements and achieve a multimode operation. Another key feature is that compared to the transmitters based on quadrature or polar modulation, the transmitter reduces the efficiency much more slowly as the output power backs off from the maximum level. Thus, the HQPM-based transmitter can achieve an optimum average-efficiency performance in application to a multimode wireless system with various dynamic output power levels.

\section{SYSTEM OVERVIEW AND COMPARISON}

Fig. 2 illustrates the detailed block diagram of the linear transmitter applying the proposed HQPM technique. There are two major components in the system. One is the switching-mode PA which is realized as a Class-E design. The other is the HQPM, with key digital blocks shown within the shadow area. The HQPM includes a digital baseband processor, a quadrature modulator and a Class-S modulator. The digital baseband processor mainly includes an envelope calculator, a predistorter, a delta-sigma modulator (DSM) and a delay unit. The envelope calculator is based on a coordinate rotation digital computer (CORDIC) unit, aiming to extract the envelope from the baseband pulse-shaped IQ signal in the digital domain. The predistorter is for compensating the PA AM-AM distortion due to the above-mentioned double envelope modulation. The DSM is to replace the conventional pulsewidth modulator for driving the Class-S modulator. The delay unit is used to compensate the delay mismatch between phase information and amplitude information at the combination point. The quadrature modulator generates the quadrature-modulated signal that goes to the RF-input terminal of Class-E PA. The Class-S modulator converts the digital envelope signal into an analog envelope signal, which goes to the supply-voltage terminal of Class-E PA.

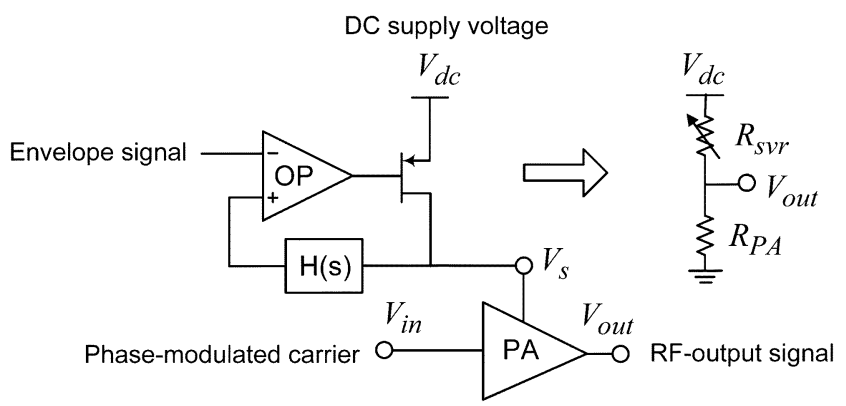

Fig. 3. Series voltage regulator applied in a polar modulator-based transmitter and its equivalent circuit.

Defining the dc-to-RF efficiency as the ratio of output RF power to input dc power for the PA along with power supply circuitry, we compare its dependence on output power in the three transmitter architectures shown in Fig. 1. Since the dc power consumption for a quadrature modulator-based transmitter is almost the same at different output power levels, the dc-to-RF efficiency is proportional to the square of the output amplitude level. This leads to an inverse relation between the normalized dc-to-RF efficiency and the output power back-off value in linear scale (OBO), which is mathematically described as

$$
\begin{aligned}
\eta & =\frac{P_{\text {out }}}{P_{\mathrm{dc}}} \propto V_{\text {out }}^{2} \Rightarrow \frac{\eta}{\eta_{\text {max }}}=\frac{V_{\text {out }}^{2}}{V_{\text {out }, \text { max }}^{2}} \\
& =\frac{P_{\text {out }}}{P_{\text {out } \text { max }}}=\mathrm{OBO}^{-1} .
\end{aligned}
$$

In a polar modulator-based transmitter, a series voltage regulator, as shown in Fig. 3, is applied to modulate the supplyvoltage terminal of the switching-mode PA [3], [4]. Such a series voltage regulator causes power dissipation in the modulation process, which can be explained using the equivalent circuit in Fig. 3. The operational amplifier (OP) adjusts the conductance of the pMOS that is in series with the supply-voltage terminal of the switching-mode PA, such that the supply voltage can track the envelope signal at the OP's input. The pMOS can be regarded as a variable resistor $\left(R_{\mathrm{svr}}\right)$ in series with a constant resistor $\left(R_{\mathrm{PA}}\right)$, which has a value equal to the equivalent resistance of the PA. The variation of $R_{\mathrm{svr}}$ is to regulate the voltage across the $R_{\mathrm{PA}}$ into the desired output amplitude, $V_{\text {out }}$. Therefore, the dc-to-RF efficiency in relation to $V_{\text {out }}$ and OBO for the polar modulator-based transmitter can be derived as

$$
\begin{aligned}
\eta & =\frac{P_{\mathrm{out}}}{P_{\mathrm{dc}}}=\frac{R_{\mathrm{PA}}}{R_{\mathrm{PA}}+R_{\mathrm{svr}}}=\frac{V_{\mathrm{out}}}{V_{\mathrm{dc}}} \\
& \Rightarrow \frac{\eta}{\eta_{\max }}=\mathrm{OBO}^{-0.5} .
\end{aligned}
$$

In the proposed HQPM-based transmitter, the dc-to-RF efficiency is equal to the product of the Class-S modulator and the switching-mode PA's conversion efficiency that is denoted by $\eta_{s}$ and $\eta_{e}$, respectively. Basically, both efficiencies are ideally irrelevant to the output amplitude level. Therefore, the dc-to-RF is ideally a constant regardless of output power level. That is

$$
\begin{aligned}
\eta & =\frac{P_{\text {out }}}{P_{\mathrm{dc}}}=\frac{P_{s}}{P_{\mathrm{dc}}} \cdot \frac{P_{\mathrm{out}}}{P_{s}}=\eta_{s} \cdot \eta_{e} \propto V_{\mathrm{out}}^{0} \\
& \Rightarrow \frac{\eta}{\eta_{\max }}=\mathrm{OBO}^{0} .
\end{aligned}
$$




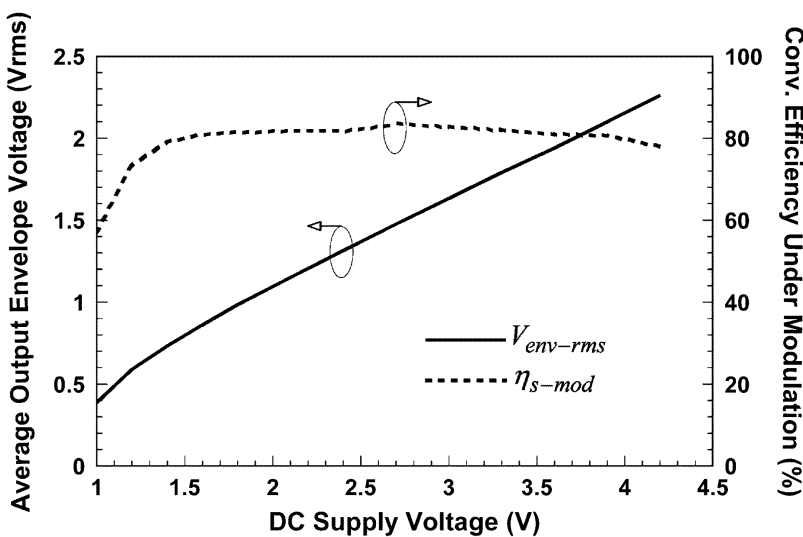

Fig. 4. Average output envelope in root mean square voltage and conversion efficiency under modulation for the Class-S modulator delivering a CDMA2000 $1 \times$ QPSK-modulated envelope signal.

Comparison of the above expressions in (1)-(3) indicates that the HQPM-based transmitter has a huge advantage over the quadrature or polar modulator-based transmitter on the efficiency performance while generating an output RF signal with a time-varying envelope under back-off conditions.

\section{SYSTEM COMPONENTS}

\section{A. Class-S Modulator}

Class-S modulators [10], also known as Class-S amplifiers or $\mathrm{dc}-\mathrm{dc}$ converters, are crucial components in envelope-tracking architectures [3] for efficiently modulating PA supply voltage in order to reduce de power consumption at low output power levels. The input envelope signal is first converted into a binary signal. In this conversion process, quantization noise inevitably contaminates the output signal and must be modulated away from the desired signal band. A first-order DSM was selected for this task because of its simple architecture and its capacity to operate at a high sampling rate for reducing the in-band quantization noise. A DSM is better than the pulsewidth modulator commonly used in Class-S modulator because its output-stage waveform recovery circuitry can be easily realized as a low-pass filter rather than an integrator. In the subsequent process, the buffer stage efficiently amplifies the binary signal and the low-pass filter converts the binary signal into an analog signal while suppressing the out-band quantization noise. This efficiently generates a low-distortion envelope signal used as the supply voltage of the switching-mode PA.

The Class-S modulator implemented in this study includes a field-programmable gate array (FPGA) for the DSM, a CMOS chip for the buffer stage, and a discrete component for the lowpass filter. Fig. 4 shows the average output envelope in root mean square voltage $\left(V_{\text {env-rms }}\right)$ and the conversion efficiency under modulation $\left(\eta_{s-\bmod }\right)$ versus the dc supply voltage $\left(V_{\mathrm{dc}}\right)$. Note that in this measurement the Class-S modulator output signal is a CDMA2000 $1 \times$ quadrature phase-shift key (QPSK)modulated envelope signal and the conversion efficiency under modulation is defined as

$$
\eta_{s-\bmod }=\frac{V_{\mathrm{env}-\mathrm{rms}}^{2}}{V_{\mathrm{dc}} I_{\mathrm{dc}} R_{L}}
$$

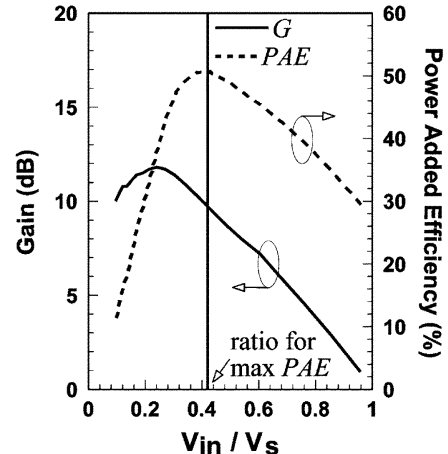

(a)

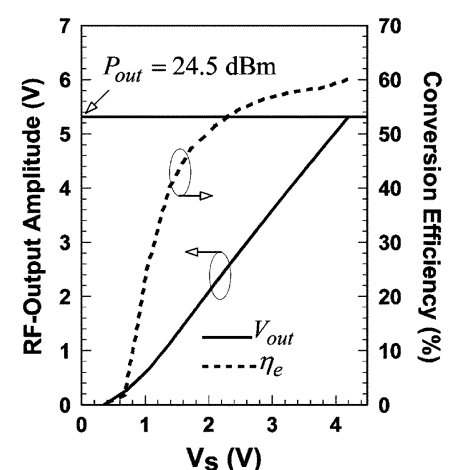

(b)
Fig. 5. CW measurement results for the Class-E PA. (a) Under a fixed supply voltage $\left(V_{s}=3.3 \mathrm{~V}\right)$. (b) Under a variable supply voltage in proportion to the input RF amplitude $\left(V_{\text {in }}=0.42 V_{s}\right)$.

where $I_{\mathrm{dc}}$ represents the dc current consumption measured at the dc supply-voltage terminal of the Class-S modulator, and $R_{L}$ is the equivalent resistance looked from the supply-voltage terminal of the switching-mode PA. Fig. 4 indicates that the conversion maintains about $80 \%$ efficiency for a dc supply voltage larger than $1.5 \mathrm{~V}$. This efficiency is a primary factor in determining the dc-to-RF efficiency of the HQPM-based transmitter.

\section{B. Class-E PA}

A switching-mode PA that adopts Class-E design is used in the proposed HQPM-based transmitter for the purpose of achieving a high average transmit efficiency. However, there are some design considerations for a Class-E PA when used with the supply-voltage modulation in this specific application. The following discussion addresses these considerations in providing a design solution for an integrated Class-E PA.

The design solution for a Class-E PA with finite dc-feed inductance was first introduced by Zulinski and Steadman [11]. Avratoglou et al. solved the problem using Laplace transforms for Class-E PAs having an arbitrary Q factor output load network [12]. Kazimierczuk et al. [13] and Klehn et al. [14] considered finite dc-feed inductance and switch current fall time. All the methods mentioned above require iterative calculations. In this research, the authors derived a closed-form solution for Class-E PAs that simultaneously considered the dc-feed inductance, switch-on resistance and package bondwire inductance in a practical fabrication process. According to the derived analytical solution, a $1.95-\mathrm{GHz}$ Class-E PA monolithic microwave integrated circuit (MMIC) was implemented in a GaAs heterojunction bipolar transistor (HBT) process.

Fig. 5(a) shows the measured gain and PAE with respect to the ratio of $V_{\text {in }}$ to $V_{s}$ by sweeping the input continuous-wave $(\mathrm{CW})$ power under a fixed supply voltage of $3.3 \mathrm{~V}$. One can see that the PA MMIC exhibits strong nonlinearity because of an acute variation in gain response. The corresponding PAE has a maximum value of $51 \%$ at a $V_{\text {in }}-$ to $-V_{s}$ ratio of 0.42 . This ratio was used for setting the supply voltage proportional to the input RF amplitude for the HBT Class-E PA applied to HQPM architecture. As shown in the measurement results of Fig. 5(b), such a variable supply voltage results in a good linear relation between $V_{s}$ and $V_{\text {out }}$. Actually, the $V_{s}-V_{\text {out }}$ relation 


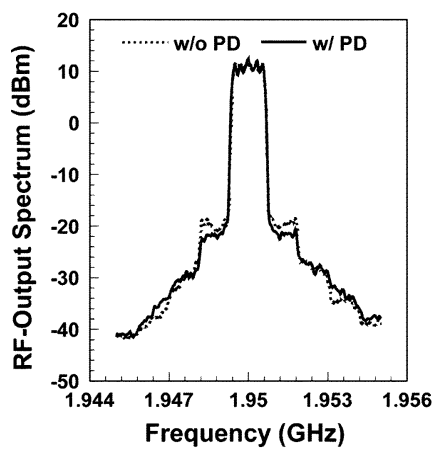

(a)

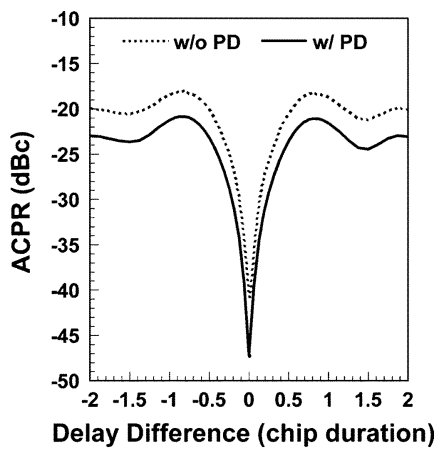

(b)
Fig. 6. Comparisons of the measured performance results for the HQPM-based transmitter with and without applying the digital predistortion scheme. (a) Output spectrum. (b) ACPR versus delay difference.

in Fig. 5(b) is not as linear at a low supply voltage as it is at a high supply voltage. This is because the HBT cannot switch on and off properly when the applied input RF amplitude that is proportional to the supply voltage is at a low level.

\section{Digital Predistorter and Delay Unit}

In Fig. 5(b), the Class-E PA's $V_{s}-V_{\text {out }}$ measurement, characterized as $f\left(V_{s}\right)$, indicates that the relation between supply voltage and RF-output amplitude is not so linear, especially at low supply voltage levels. This research proposes a digital predistorter for compensating the nonlinear $V_{s}-V_{\text {out }}$ relation. A predistortion function, $g\left(V_{s}\right)$, is given for producing the predistorted supply voltage $\left(V_{s}^{\prime}\right)$ to make the corresponding RF-output amplitude $\left(V_{\text {out }}^{\prime}\right)$ maintain a linear relation with $V_{s}$. Mathematically, the linearization relation for the Class-E PA can be written as

$$
V_{\text {out }}^{\prime}\left(V_{s}\right)=f\left(g\left(V_{s}\right)\right)=f\left(V_{s}^{\prime}\right)=k \cdot\left(V_{s}-V_{\text {knee }}\right)
$$

where $k$ is a constant related to a targeted linear gain given as

$$
G=\frac{V_{\mathrm{out}}^{\prime}}{V_{\mathrm{in}}}=\frac{k}{r_{v}} .
$$

In (5), $V_{\text {knee }}$ is a knee voltage of $0.35 \mathrm{~V}$ in a GaAs HBT. Also note that $r_{v}$ in (6) denotes the constant $V_{\mathrm{in}}$-to- $V_{s}$ ratio applied to the Class-E PA. By referring to Fig. 5(a) and (b), $r_{v}$ and $G$ are selected as 0.42 and $3.28(10.3 \mathrm{~dB})$, respectively. The value of $k$ is calculated equal to 1.38 .

From (5), the predistortion function, $g\left(V_{s}\right)$, can be derived as the inverse function of $f\left(V_{s}\right)$ and then curve-fitted as a polynomial. The digital predistorter is implemented as a digital arithmetic unit synthesized according to the curve-fitted polynomial. Fig. 6(a) compares the measured output spectra with and without using the digital predistorter for the HQPM-based transmitter applied with a CDMA2000 $1 \times$ QPSK-modulated signal. The average modulated output power is at $8 \mathrm{~dB}$ back-off from the maximum $\mathrm{CW}$ output power. The comparison shows that the digital predistorter improves the adjacent channel power ratio (ACPR) by about $6.3 \mathrm{~dB}$.

The delay unit is constructed by a stack of registers, and compensates the delay difference between the envelope and quadrature-modulated signal paths. For the same case as presented in

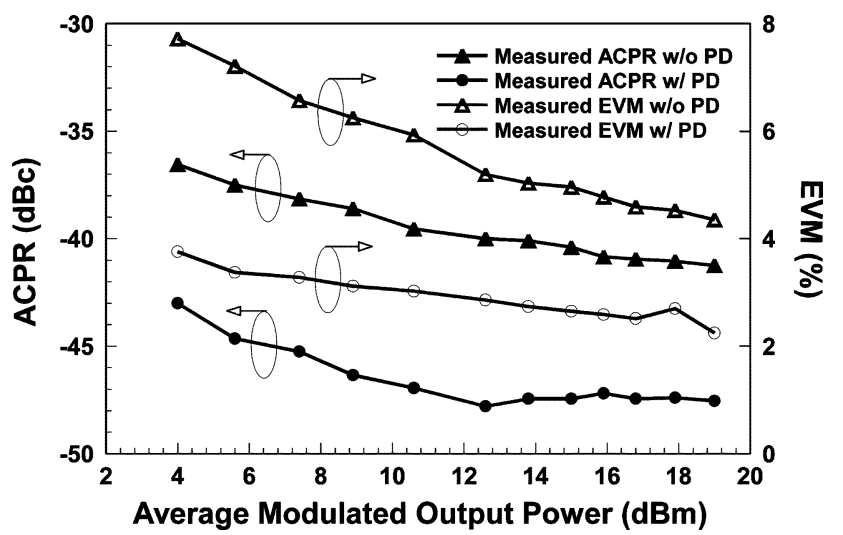

Fig. 7. Comparisons of measured ACPRs and EVMs for the implemented HQPM-based transmitter with and without applying the digital predistortion scheme.

Fig. 6(a), the measured ACPRs accounting for the delay mismatch effects are shown in Fig. 6(b). It is witnessed that the HQPM-based transmitter is sensitive to the delay difference, especially in the interval between -0.5 and 0.5 chip durations.

\section{RESUlTS AND DisCUSSION}

For demonstration, a prototype transmitter implemented according to the proposed HQPM architecture shown in Fig. 2 generates 1.95-GHz CDMA2000 $1 \times$ QPSK-modulated signals with $1.23 \mathrm{MHz}$ modulation bandwidth. Considering that the PAPR is about $5.5 \mathrm{~dB}$ for this kind of transmit signal, the Class-E PA designed to have a maximum $\mathrm{CW}$ power of $24.5 \mathrm{dBm}$ is qualified to deliver CDMA2000 $1 \times$ signals with average modulated power up to $19 \mathrm{dBm}$. The Class-S modulator dc supply voltage varies to adjust the average modulated output power level. The Class-S modulator's buffer stage and the Class-E PA are self-designed and implemented integrated circuits (ICs), which has been described in Section III. The quadrature modulator and digital-analog converters (DACs) rely on commercial ICs. The baseband processor is realized using FPGA. The predistorted envelope's baseband and DSM signal has a sampling frequency equal to 8 and 64 times the chip rate $(1.2288 \mathrm{MHz})$.

The measured results for ACPR and error vector magnitude (EVM) are demonstrated in Fig. 7. The results with and without using the digital predistorter are also shown for comparison. In Fig. 7, both ACPR and EVM decrease with increasing output power levels. This is because the Class-E PA's $V_{s}-V_{\text {out }}$ relation is more linear at higher supply-voltage levels. From the measurement results, the digital predistorter improves the ACPR by 2 to $7 \mathrm{~dB}$, and the EVM by $2 \%$ to $4 \%$ in the measured output power range from 4 to $19 \mathrm{dBm}$. It is noted that better improvement for both ACPR and EVM can be expected if the digital predistortion further considers AM-PM and memory effects.

The overall efficiency of the HQPM-based transmitter is determined by the conversion efficiency of the Class-E PA and the Class-S modulator. The transmitter's dc-to-RF efficiency in dealing with the constant-envelope modulated signals equals the product of these two component efficiencies. However, the transmitter's dc-to-RF efficiency in dealing with the time-varying modulated signals also depends on the signal distribution at various instantaneous envelope levels. From the measurement results shown in Fig. 5(b), the output power and 


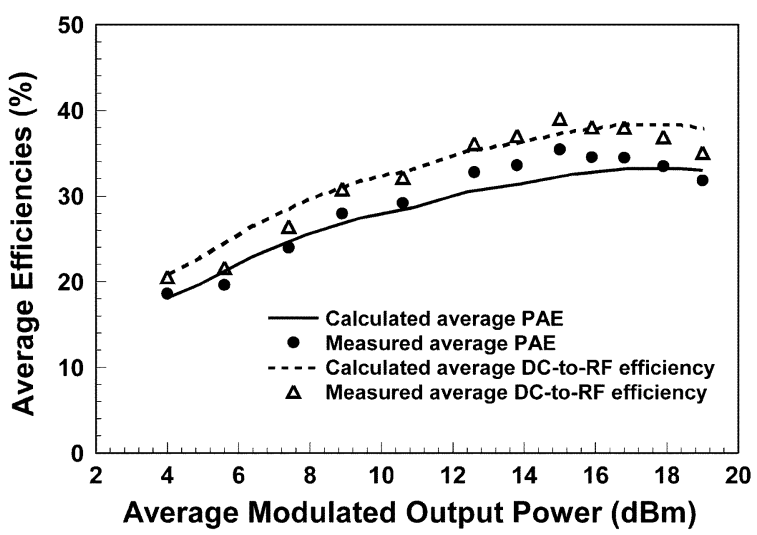

Fig. 8. Comparisons of calculated and measured average efficiencies for the implemented HQPM-based transmitter delivering 1.95-GHz CDMA2000 $1 \times$ QPSK-modulated signals.

the conversion efficiency of the Class- $E$ PA can be found and characterized as $P_{\text {out }}\left(V_{s}\right)$ and $\eta_{e}\left(V_{s}\right)$, respectively, where $V_{s}$ represents the instantaneous envelope voltage fed to the Class- $E$ PA supply-voltage terminal. The Class-S modulator's conversion efficiency under modulation is shown in Fig. 4 and simply expressed here as $\eta_{s-\bmod }\left(V_{\mathrm{sp}}\right)$ where $V_{\mathrm{sp}}$ represents the peak value of $V_{s}$ and is equivalent to the Class-S modulator dc supply voltage. Note that $\eta_{s-\bmod }\left(V_{\mathrm{sp}}\right)$ actually represents an average conversion efficiency for the Class-S modulator delivering a CDMA2000 $1 \times$ QPSK-modulated envelope signal with an instantaneous level varying within a range from 0 to $V_{\mathrm{sp}}$. Assume that the probability of an instantaneous envelope level is characterized as $p\left(V_{s}\right)$. With the functions defined above, the average dc-to-RF efficiency for the HQPM-based transmitter can be found as

$$
\eta_{\text {avg }}=\eta_{s-\bmod }\left(V_{\mathrm{sp}}\right) \frac{\int_{0}^{V_{\mathrm{sp}}} P_{\mathrm{out}}\left(V_{s}\right) \cdot p\left(V_{s}\right) d V_{s}}{\int_{0}^{V_{\mathrm{sp}}} \frac{P_{\text {out }}\left(V_{s}\right) \cdot p\left(V_{s}\right)}{\eta_{e}\left(V_{s}\right)} d V_{s}} .
$$

The corresponding average PAE can be calculated as

$$
\mathrm{PAE}_{\mathrm{avg}}=\eta_{\mathrm{avg}}\left(1-\frac{1}{G^{2}}\right)
$$

where $G$ is the selected constant gain expressed in (6).

Fig. 8 compares the calculated average dc-to-RF efficiency and average PAE from (7) and (8), respectively, with the measured results. The comparisons show good agreement. The average dc-to-RF and PAE efficiency ranges from $21 \%$ to $39 \%$ and from $19 \%$ to $36 \%$, respectively, in the entire measurement output power range. These results can evidence that the im- plemented HQPM-based transmitter drops in efficiency quite slowly during the power back-off.

\section{CONCLUSION}

The presented HQPM-based transmitter that uses a Class-E PA has been identified as having great potential for application to a multimode wireless system with various data rates, output power levels, and digital modulations. The high performance for the transmitter is attributed to the fact that the applied predistorted envelope and quadrature-modulated signals can effectively linearize the Class-E PA with high efficiency over a large output power range.

\section{REFERENCES}

[1] S. Forestier, P. Bouysse, R. Quere, A. Mallet, J. M. Nebus, and L. Lapierre, "Joint optimization of the power-added efficiency and the error-vector measurement of 20-GHz pHEMT amplifier through a new dynamic bias-control method," IEEE Trans. Microw. Theory Tech., vol. 52, no. 4, pp. 1132-1141, Apr. 2004.

[2] D. Junxiong, P. S. Gudem, L. E. Larson, D. F. Kimball, and P. M. Asbeck, "A SiGe PA with dual dynamic bias control and memoryless digital predistortion for WCDMA handset applications," IEEE J. SolidState Circuits, vol. 41, no. 5, pp. 1210-1221, May 2006.

[3] J. Staudinger, B. Gilsdorf, D. Newman, G. Norris, G. Sadowniczak, R. Sherman, and T. Quach, "High efficiency CDMA RF power amplifier using dynamic envelope tracking technique," in Dig. IEEE MTT-S Int. Microw. Symp., 2000, pp. 873-876.

[4] F. Wang, A. H. Yang, D. F. Kimball, L. E. Larson, and P. M. Asbeck, "Design of wide-bandwidth envelope-tracking power amplifiers for OFDM applications," IEEE Trans. Microw. Theory Tech., vol. 53, no. 4, pp. 1244-1255, Apr. 2005.

[5] P. Reynaert and M. S. J. Steyaert, "A 1.75-GHz polar modulated CMOS RF power amplifier for GSM-EDGE," IEEE J. Solid-State Circuits, vol. 40, no. 12, pp. 2598-2608, Dec. 2005.

[6] A. W. Hietala, "A quad-band 8PSK/GMSK polar transceiver," IEEE J. Solid-State Circuits, vol. 41, no. 5, pp. 1133-1141, May 2006.

[7] L. R. Kahn, "Single sideband transmission by envelope elimination and restoration," Proc. IRE, vol. 40, no. 7, pp. 803-806, Jul. 1952.

[8] J.-K. Jau, Y.-A. Chen, S.-C. Hsiao, T.-S. Horng, and J.-Y. Li, "Highly efficient multimode RF transmitter using the hybrid quadrature polar modulation scheme," in Dig. IEEE MTT-S Int. Microw. Symp., 2006, pp. 789-792.

[9] C.-J. Li, T.-S. Horng, J.-K. Jau, and J. Y. Li, "System design issues in a HQPM-based transmitter," in Dig. IEEE MTT-S Int. Microw. Symp., 2007, pp. $77-80$

[10] F. H. Raab and D. J. Rupp, "Class-S high efficiency amplitude modulator," RF Design, vol. 17, no. 5, pp. 70-74, May 1994.

[11] R. E. Zulinski and J. K. Steadman, "Class E power amplifiers and frequency multipliers with finite dc-feed inductance," IEEE Trans. Circuits Syst., vol. CAS-34, no. 9, pp. 1074-1087, Sep. 1987.

[12] C. P. Avratoglou, N. C. Voulgaris, and F. I. Ioannidou, "Analysis and design of a generalized class E tuned power amplifier," IEEE Trans. Circuits Syst., vol. 36, no. 8, pp. 1086-1079, Aug. 1989.

[13] M. Kazimierczuk and K. Puczko, "Exact analysis of class E tuned power amplifier at any Q and switch duty cycle," IEEE Trans. Circuits Syst., vol. CAS-34, no. 2, pp. 149-159, Feb. 1987.

[14] B. E. Klehn and S. S. Islam, "An exact analysis of class-E power amplifiers for RF communications," in Proc. IEEE Int. Symp. Circuits Syst., 2004 , pp. $13-26$. 